\title{
A Formal Model for Forensic Storage Media Preparation Tools
}

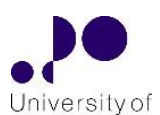

Portsmouth

\section{Benjamin Aziz}

benjamin.aziz@port.ac.uk School of Computing, University of Portsmouth, United Kingdom
Philippe Massonet, Christophe Ponsard

\{philippe.massonet,christophe.ponsard\}@cetic.be CETIC, Charleroi Belgium

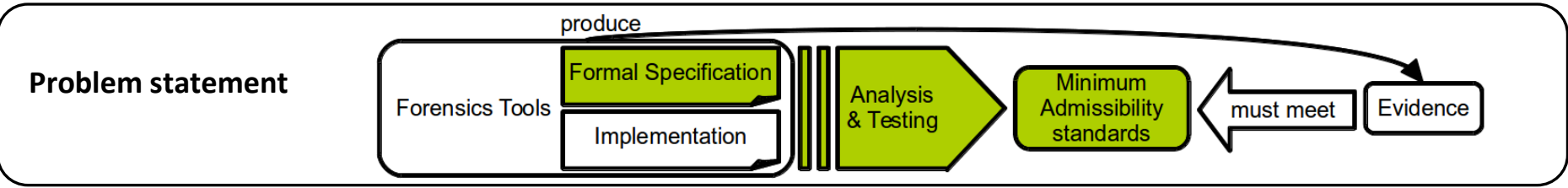

Event-B formal language

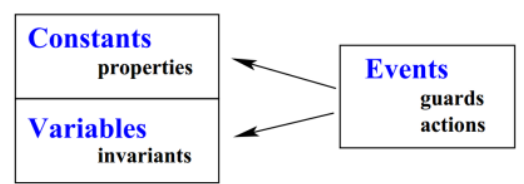

Dynamic Part

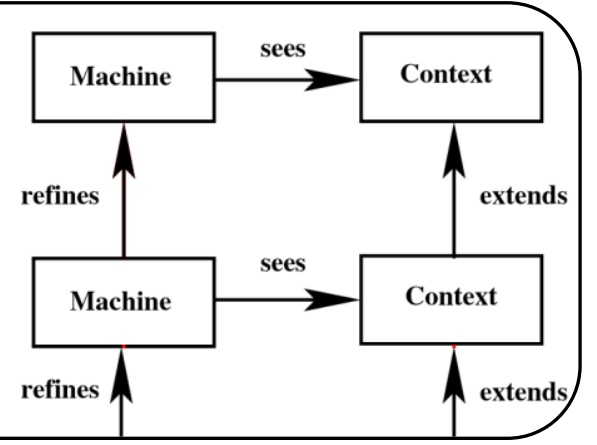

CONTEXT SETS

ForensicsStorageMedia

Data

CONSTANTS

benignDataElement

AXIOMS

$\operatorname{axm} 3:$ Data $\neq \varnothing$

axm2 : benignDataElement $\in$ Data END

MACHINE VisibleSectors SEES ForensicsStorageMedia

VARIABLES

ForensicStorageMedium

Terminated

INVARIANTS

inv1: $\quad$ ForensicStorageMedium $\subseteq$ Data

inv2:

Terminated $\in$ BOOL

Terminated $=$ TRUE $\Rightarrow$

\section{Completeness : $\forall \mathbf{\forall} \cdot(\mathbf{x} \in$ ForensicStorageMedium $)$} $\Rightarrow(\mathrm{x}=$ benignDataElement $))$

EVENTS

ANY

Preparation $\hat{=}$

visibleSector

benignDataSet

WHERE

grd1 : Terminated $=$ FALSE

grd2 : visibleSector $\subseteq$ ForensicStorageMedium

$\operatorname{grd} 3: \forall \mathrm{x} \cdot(\mathrm{x} \in$ visibleSector $) \Rightarrow(\mathrm{x} \neq$ benignDataElement $)$

$\operatorname{grd} 4: \quad$ visibleSector $\neq \varnothing$

grd5: $\forall \mathrm{x} \cdot(\mathrm{x} \in$ benignDataSet $) \Rightarrow(\mathrm{x}=$ benignDataElement $)$

grd6 : $\quad \operatorname{card}($ benignDataSet $)=$ card $($ visibleSector $)$

THEN

ForensicStorageMedium :=

act1 : ((ForensicStorageMedium \visibleSector) $U$ benignDataSet)

END
MACHINE WithHiddenSectors REFINES VisibleSectors

VARIABLES

ForensicStorageMediumHiddenAreas

overwriteHiddenData // support overwriting hidden areas

INVARIANTS

inv1: ForensicStorageMediumHiddenAreas

$\subseteq$ Data

inv2 : overwriteHiddenData $\in$ BOOL

EVENTS

ANY

HiddenSectorPreparation $\hat{=}$

hiddenSector

benignDataSet

WHERE

grd7 : $\quad$ Terminated $=$ FALSE

grd8 : hiddenSector $\subseteq$ ForensicStorageMediumHiddenAreas

grd9 : $\forall \mathrm{x} \cdot(\mathrm{x} \in$ hiddenSector $) \Rightarrow(\mathrm{x} \neq$ benignDataElement $)$

$\operatorname{grd} 10:$ hiddenSector $\neq \varnothing$

grd11 : $\forall x \cdot(x \in$ benignDataSet $\Rightarrow(x=$ benignDataElement $)$

$\operatorname{grd12}: \quad \operatorname{card}($ benignDataSet $)=$ card(hiddenSector)

grd13 : overwriteHiddenData $=$ TRUE

THEN

ForensicStorageMediumHiddenAreas :=

((ForensicStorageMediumHiddenAreas

$\backslash$ hiddenSector) $\cup$ benignDataSet)

\section{END}

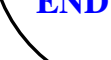

Discussion: a formal reference modelling using a refinement approach (using Event-B here) enables

1. the progressive injection of details into the model as needed by the system and context (including threats) e.g. an additional third level can be introduced for the Erase hardware command in the prepared device.

2. specific proofs to be established w.r.t. tool (design) properties such as correctness but also completeness however accuracy could not be specified or reasoned on in a uniform manner

Key References: Abrial, J.-R. (2010). Modeling in Event-B: System and Software Design. Cambridge University Press Casey, E. and Rose, C. (2010). Forensic Discovery: Handbook of Digital Forensics and Investigation. Academic Press. Gladyshev, P. and Enbacka, A. (2007). Rigorous Development of Automated Inconsistency Checks for Digital Evidence Using the B Method. International Journal of Digital Evidence, 6(2). 